\title{
RÉPONSE PHYSIOPATHOLOGIQUE DES CORPUSCULES DE STANNIUS DU GARDON, RUTILUS rUTILUS, A DES PERTURBATIONS DE L'ENVIRONNEMENT
}

\author{
Evelyne LOPEZ, J.C. LUCHETTA*, Jacqueline PEIGNOUX, \\ avec la collaboration technique de Bernadette VIDAL et Francine LALLIER
}

Laboratoire de Physiologie générale et comparée, C.N.R.S. URA 90, Muséum national d'Histoire naturelle, 7, rue Cuvier, 75005 PARIS.

\footnotetext{
* Conseil Supérieur de la Pêche, délégation régionale de Compiègne,

3,rue Ste-Marie, 60200 COMPIEGNE.
}

\section{RÉSUMÉ}

Les corpuscules de Stannius (CS), glandes spécifiques des téléostéens, présentent des altérations de structure et d'activité en réponse à un environnement modifié.

Nous avons étudié les CS de Gardons du Canal à Grand Gabarit du Nord/Pas-de-Calais prélevés sur quatre stations, choisies en raison des composantes physicochimiques du milieu. Sur l'une d'entre elles, où les taux de nitrites $\left(\mathrm{NO}_{2}\right)$ et d'ammonium $\left(\mathrm{NH}_{4}\right)$ sont élevés, nous avons mis en évidence une hyperactivité de ces glandes très anormale et significative en comparaison de l'état constaté aux mêmes saisons dans un milieu peu pollué (normes de la CEE). En un point où le milieu environnant est plus dégradé (taux de $\mathrm{NO}_{2}$ et $\mathrm{NH}_{4}$ particulièrement élevés), les CS montrent un aspect pathologique qui peut conduire, dans certaines conditions plus sévères, à des phénomènes de dégénérescence de la glande. Les CS sont des indicateurs sensibles des variations de composition de l'environnement.

\section{PHYSIOPATHOLOGICAL RESPONSE OF STANNIUS CORPUSCLES OF THE ROACH, RUTILUS RUTILUS, TO DISTURBANCES OF THE ENVIRONMENT}

\section{SUMMARY}

Corpuscles of Stannius (CS) which are specific glands of teleosteans show alterations of structure and activity in response to changes in environment.

We have studied Stannius corpuscles of roachs in the "Canal à Grand Gabarit du Nord/Pasde-Calais". They were taken in four stations selected because of the physicochemical components of the environment.

In one of the station, where the concentration of nitrites $\left(\mathrm{NO}_{2}\right)$ and ammonium $\left(\mathrm{NH}_{4}\right)$ are more important than the values fixed by CEE, we have shown an abnormal hyperactivity of these glands, in contrast to the CS histological aspect observed in roach living in a control site. In a region presenting a critical situation, the environment being particularly modified, the CS presents a pathological feature leading in some drastic conditions to a degeneration of the gland. CS are sensible indicators of variations of the composition of the environment.

\section{INTRODUCTION}

Le comportement des différentes espèces piscicoles rencontrées dans nos rivières, leur variété, leur répartition, leur dynamique de reproduction, leur aspect extérieur ainsi que leur qualité comestible et gastronomique sont le reflet de l'état de leur biotope.

Tous ces paramètres dépendent de leur état physiologique. Afin d'évaluer certains as pects de celui-ci, nous avons étudié les corpuscules de Stannius (glandes spécifiques des poissons téléostéens) pour les raisons suivantes: chez les poissons, la principale interface entre milieu intérieur et milieu extérieur est la branchie qui assure le rôle physiologique primordial notamment dans les transferts d'ions et en particulier du calcium. Par ailleurs, les corpuscules de Stannius (CS) sécrètent I'hormone régulant les flux de Ca branchiaux (FONTAINE, 1964, 1967; MILET et al., 1979). Il a été démontré lors d'études écotoxicologiques antérieures que la branchie est tout particulièrement touchée en cas de contaminations importantes du milieu extérieur (HAWKES, 1977; LOPEZ et al., 
1981 a, b ; CRESPO et al., 1981 ; DAOUST et al., 1984 ; KARLSSON-NÖRRGREN et al., 1985, 1986 ; MALLET, 1985; PEREIRA, 1988), ce qui entraîne des modifications de la structure et de l'activité des CS (MARTOJA et al., 1984 ; LOPEZ et al., 1985) rapidement décelables et indicatrices de perturbations de l'environnement.

Des "pollutions accidentelles" avec mortalité piscicole spectaculaire sont souvent observées dans le canal à Grand Gabarit du Nord/Pas-de-Calais. Une étude sur ce site a èté envisagée dès 1984 (en collaboration avec le Conseil Supérieur de la Pêche de Compiègne et l'Agence de l'Eau Artois-Picardie) et le Gardon "Rutilus rutilus" a été choisi comme poisson de référence pour sa forte résilience.

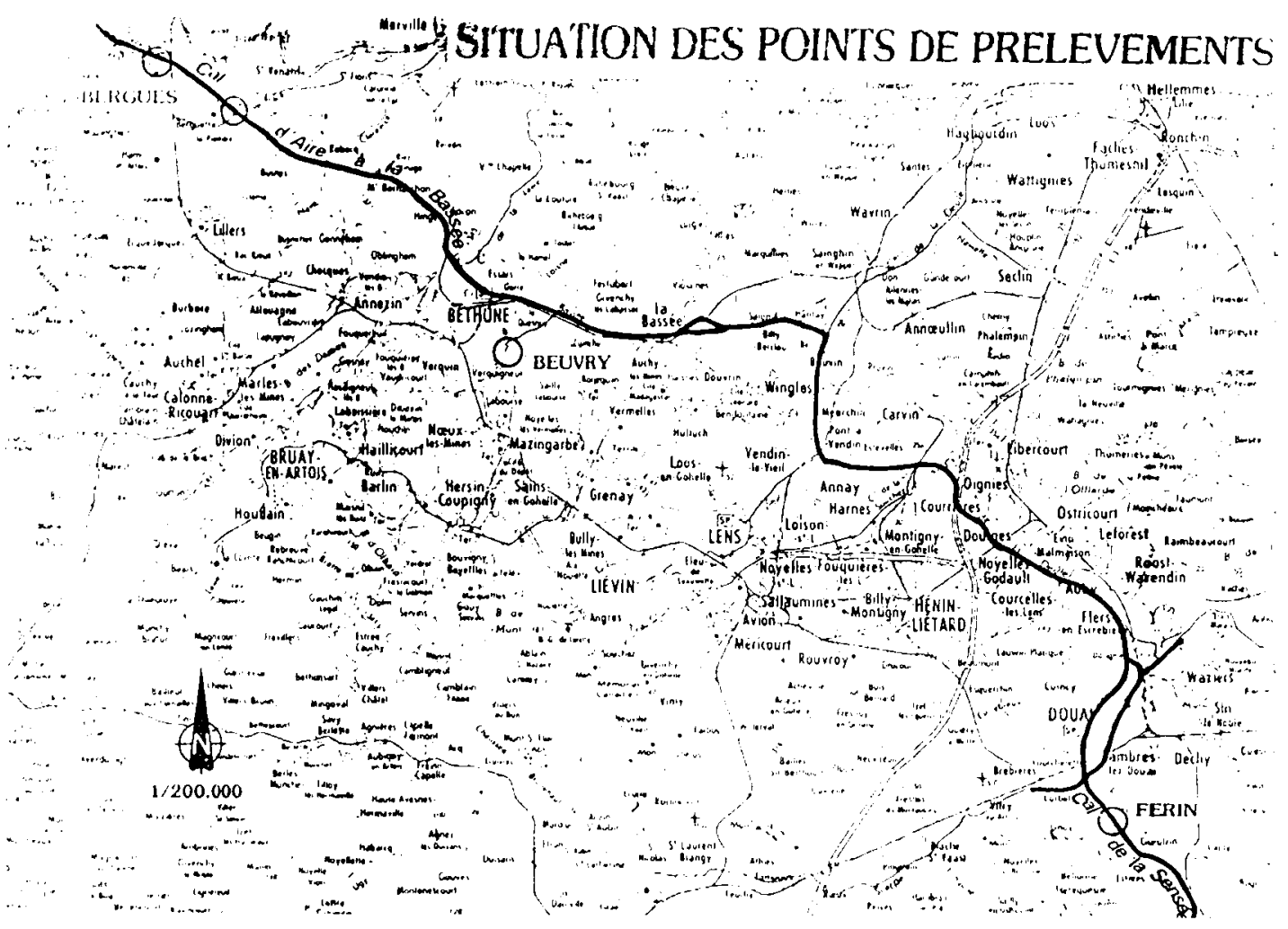

figure 1 : Situation des points de prélèvements.

figure 1 : Situation of the four selected stations.

\section{MATÉRIEL ET MÉTHODES}

\section{Sites étudiés et raisons du choix}

Quatre sites ont été choisis : Férin sur le canal de la Sensée, Beurry sur le canal de La Bassée et Isbergues sur le canal de La Bassée en amont et en aval d'Usinor (fig. 1).

Le cours du canal à Grand Gabarit du Nord/Pas-de-Calais est suffisamment uniforme, tant par son faciès que par ses composantes morphodynamiques, pour étudier en divers points l'influence sur les poissons d'un seul facteur variable: la qualité de l'eau. Ainsi des paramètres tels que le débit, la vitesse du courant, la température, les berges, les caches et abris qui ont une incidence importante sur le comportement des poissons sont dans cet écosystème constants sur tous les points.

Les sites précités ont été choisis pour évaluer plus particulièrement l'influence des rejets urbains et/ou industriels. Férin situé en amont de l'agglomération de Lens et de ses pôles industriels est considéré comme site de référence; Beuvry est soumis à l'influence de l'agglomération lensoise et des usines environnantes: les deux points situés en amont et aval d'lsbergues encadrent le groupe industriel Usinor. 
Les principaux indicateurs de la qualitè de l'eau, fournis par l'Agence de l'Eau Artois-Picardie, sont résumés dans le Tableau 1.

Tableau I : Composantes physicochimiques de l'eau (mg/l)

Table I : Physichochemical components of water $(\mathrm{mg} / \mathrm{l})$.

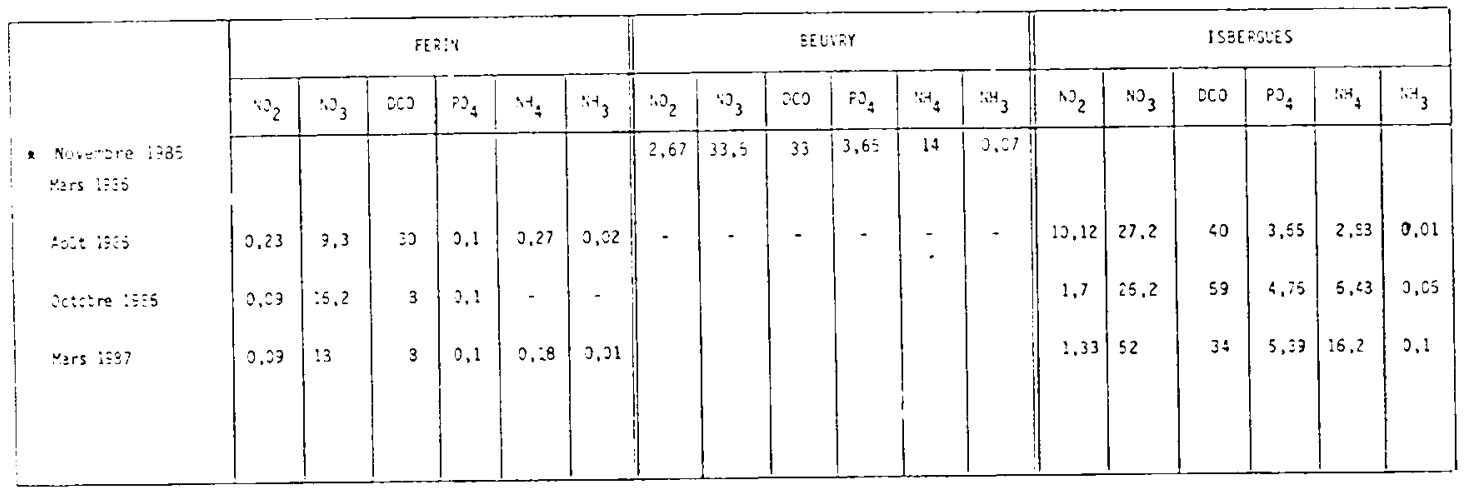

* Sart portees ici les seuies cornees dont on dispose sur ce site.

\section{Matériel et collecte des échantillons}

Quatre séries de sondages ichtyologiques ont été effectuées par pêche électrique dans les quatre stations indiquées (fig. 1), en mars, août et octobre 1986 puis en mars 1987 ; soit au total 16 sondages correspondant à différentes époques de l'année caractérisées par une évolution de l'écosystème (yoir Tableau II).

Tableau II : Matériel piscicole obtenu (Rutllus rutllus).

Table II : Collected Fish (Rutilus rutilus).

\begin{tabular}{|c|c|c|c|c|}
\hline$\gamma^{\text {Lieu }}$ & Férin & Beuvry & & \\
\hline Mars 1986 & 8 & 0 & $\begin{array}{l}\text { Amont } \\
\text { Aval }\end{array}$ & $\begin{array}{l}1 \\
1\end{array}$ \\
\hline Aoūt 1986 & 10 & 8 & $\begin{array}{l}\text { Amont } \\
\text { Aval }\end{array}$ & $\begin{array}{l}5 \\
6\end{array}$ \\
\hline Octobre 1986 & 9 & 15 & $\begin{array}{l}\text { Amont } \\
\text { Aval }\end{array}$ & $\begin{array}{l}10 \\
16\end{array}$ \\
\hline Mars 1987 & 9 & 0 & $\begin{array}{l}\text { Arront } \\
\text { Aval }\end{array}$ & $\begin{array}{l}5 \\
0\end{array}$ \\
\hline
\end{tabular}


Les prélèvements et fixations des glandes étudiées ont dans tous les cas été pratiqués sur le terrain. Tous les poissons sur lesquels ont été faits des prélèvements étaient sains extérieurement, ils ne présentaient pas de lésions cutanées

Les récoltes (Tableau II) ont été inégales selon les sites et les saisons en dépit de l'effort soutenu de prospection.

\section{Méthodes histologiques et histochimiques}

Les reins des Gardons contenant les corpuscules de Stannius, non repé rables à l'œil nu chez cette espèce, ont été prélevés sur place sur les poissons vivants et immédiatement immergés dans le liquide de fixation (Bouin aqueux).

Les échantillons ont ensuite été traités au laboratoire selon les techniques classiques de l'histologie et inclus dans le paraplast. Les blocs ont été débités en coupes sérièes de $5 \mu \mathrm{m}$ d'épaisseur afin d'effectuer la recherche systématique des glandes. Diverses colorations ont été utilisées : hémalun-eosine; coloration trichrome de Cleveland Wolfe; acide périodique, réactif de Schiff (APS). Cette dernière coloration spécifique des protéines de cosécrétion hormonale permet de mettre en évidence la présence des grains de sécrétion dans les cellules (TISSERAND-JOCHEM et al., 1987).

\section{RESULTATS}

Les CS du Gardon apparaissent entourés d'une capsule conjonctive très fine, ils sont ainsi en contact interne avec le tissu rénal, ce qui n'est pas le cas chez tous les téléostéens.

1. Les corpuscules de Stannius des Gardons pêchés à Férin (site témoin) présentent des cellules groupées en follicules arrondis, plus ou moins réguliers, séparés par des travées de tissu conjonctif assez épaisses (fig. 2). Les cellules sont triangulaires, petites, avec une membrane cytoplasmique peu délimitée. Elles ont des noyaux ovoïdes très basophiles, dont on ne distingue pas le nucléole. Les granulations intracytoplasmiques sont fines, colorables par le bleu d'aline et APS ${ }^{+}$. Les espaces interfolliculaires et vasculaires ne sont pas dilatés et les capillaires sanguins difficilement décelables (fig.3). Les cytoplasmes sont plus clairs en août et octobre (fig. 4). Toutefois aucune différence notable d'activité n'est observée au cours des diverses périodes de l'année.

L'aspect histologique de ces glandes témoigne d'une activité physiologique non perturbée.

2. Les corpuscules de Stannius des Gardons pêchés à Beuvry (fig. 5) ont un aspect histologique différent des précédents (voir fig. 2). Nous notons un déroulement des follicules en larges cordons de cellules et une hyperhémie avec une dilatation importante des capillaires sanguins s'étendant largement entre les travées cellulaires. Les CS pré levés en août et octobre (fig. 6 et 7 ) présentent une hyperplasie cellulaire et une très forte élévation du rapport nucléoplasmique. Les noyaux sont sphériques, dilatés avec des nucléoles apparents; seuls de très rares amas de granulations intracytoplasmiques peuvent être décelés.

L'ensemble de ces observations mettent en évidence une hyperactivité glandulaire qui n'a pu être décelée chez les témoins.

\section{Les corpuscules de Stannius des Gardons pêchés à Isbergues (amont et aval) en août et octobre} (fig. $8,9,10$ ) montrent des follicules déroulés en larges cordons méandriformes, très denses, séparés par des capillaires sanguins nombreux et dilatés et dans certains cas une hyperplasie et hypertrophie cellulaire (fig. 11). Les cellules en contact avec des espaces vasculaires larges ont un pôle basal riche en grains de sécrétion. Les noyaux sont hypertrophiés, ils présentent quelquefois des figures réniformes ou plusieurs nucléoles. Les membranes cytoplasmiques sont difficilement répérables et une désorganisation dans l'orientation des cellules est apparente; certaines d'entre elles sont en voie de lyse (fig. 8, 9 et 10). Les cellules peuvent être groupées en cordons très denses, elles ont alors un cytoplasme rétracté rempli de substance très basophile, elles sont disposées de façon anarchique (fig. 10).

Les CS prélevés sur les mêmes sites, en mars 1987, apparaissent extrêmement modifiés (fig. 12), leur taille est très réduite, l'organisation des cellules groupées en follicules ou cordons a disparu: on distingue des travées de cellules très denses et méandriformes séparées par des méats dilatés, certains d'entre eux correspondent à des capillaires sanguins, d'autres à des zones de rétraction. Des régions en dégénérescence totale peuvent être observées (ex. fig. 12 et 13). Les cellules de taille très réduite ne sont plus orientées, elles sont anormalement basophiles et on ne distingue plus leurs limites cellulaires. Des débris cellulaires résultant d'une lyse sévère sont observables ainsi que de nombreux noyaux pycnotiques isolés. Certains noyaux clairs, à gros nucléoles, persistent et témoignent de la grande activité antérieure.

Ces différents aspects montrent un blocage du processus de décharge hormonale pouvant conduire à divers degrés de dégénérescence cellulaire. 
figs. 2, 3, 4 : Coupes transversales de CS prélevés sur des Gardons pêchés à la station de Férin. Coloration Cleveland Wolfe.

figs. 2, 3, 4 : Cross sections of CS of the Roach caught at the station of Ferin. Staining Cleveland Wolfe.

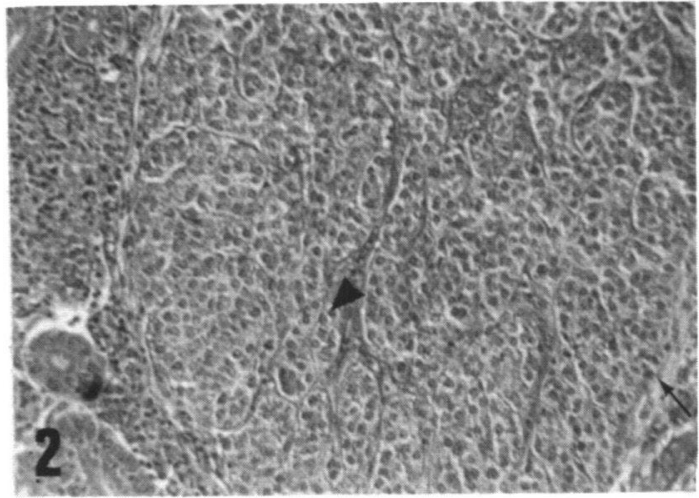

figure 2 : Thèque conjonctive très fine $(\rightarrow)$; follicules arrondis $(-)$; travées conjonctives interfolliculaires épaisses. (-.) CS au repos (Mars). G $\times 160$.

figure 2 : Thin connective theca $(\rightarrow)$; sphérical follicles $(-)$ thick connective interfollicular arches (-- ). Resting CS. (March). G x 160.

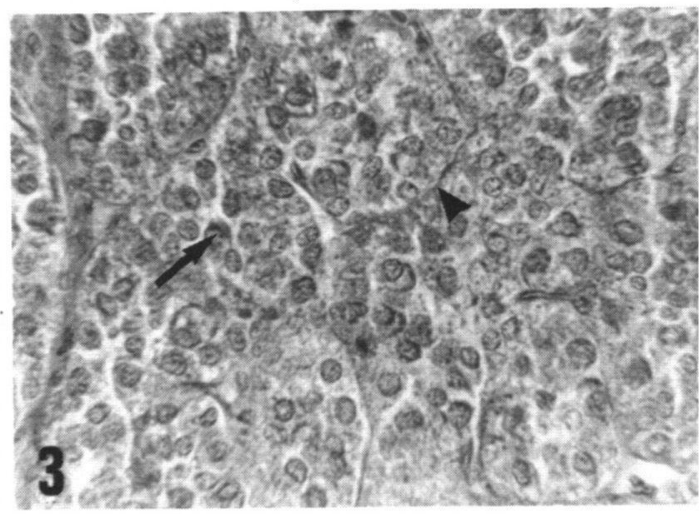

figure 3 : Cellules petites avec noyaux basophiles et granulations intracytoplasmiques $(\rightarrow)$; follicules bien délimités sans espaces vasculaires $(-)$. CS au repos (mars). $\mathbf{G} \times 400$.

figure $3 \quad$ : Little cells with basophilic nucleus and intracytoplasmic granules $(\rightarrow)$; well delimited follicles without vascular spaces ( - ). Resting CS (March). G x 400 .

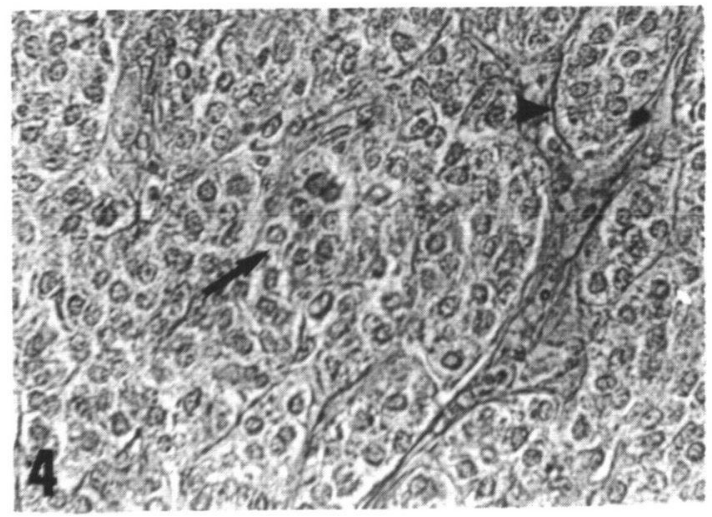

figure $4 \quad$ : Cellules petites à cytoplasmes pauvres en granulations $(\rightarrow)$ ). Travées conjonctives épaisses entre les follicules ( - ). CS au repos (octobre). G $\times \mathbf{4 0 0}$.

figure $4 \quad$ : Little cells with cytoplasm poorly granulated $(\rightarrow)$. Thick connective arches between follicles ( - ). Resting CS (October). G x 400 . 
figs. 5, 6,7 : Coupes transversales de CS prélevés sur des Gardons pêchés à Beuvry. Coloration Cleveland Wolfe.

figs. 5, 6, 7 : Cross sections of CS of the Roach caught at the station of Beuvry. Staining Cleveland Wolfe.

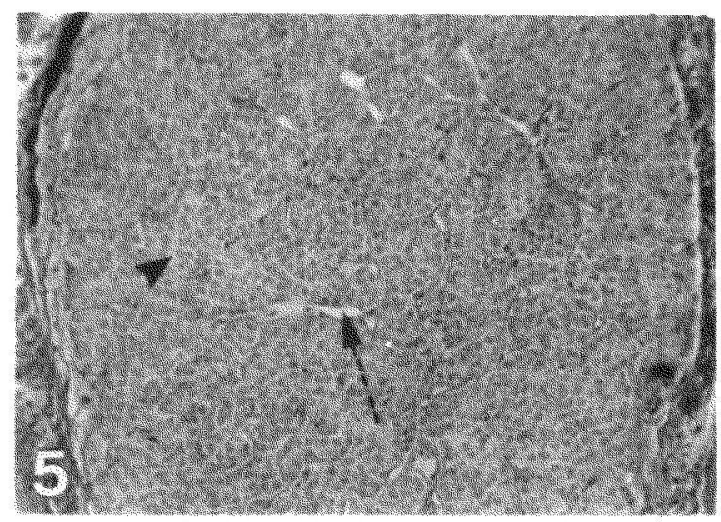

figure 5 : Follicules déroulès en larges cordons de cellules $(-)$. Vaisseaux sanguins dilatés et disparition des travées conjonctives interfolliculaires $(\ldots)$ ). CS hyperactifs. $\mathrm{G} \times 160$.

figure 5 : Large widening cellular cords $(-)$. Dilated blood vessels and disappearing of connective interfollicular arches $(-\infty)$. Hyperactive CS. G $\times 160$.

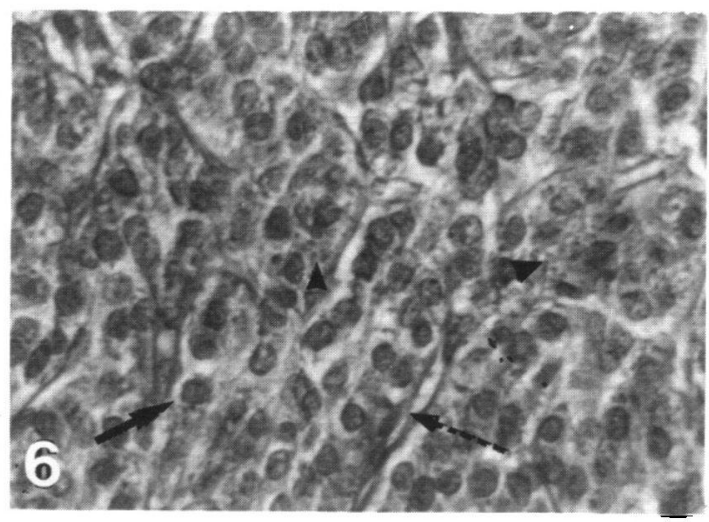

figure $6 \quad$ : Cellules dilatées avec de gros noyaux $(\rightarrow)$; granulations intracytoplasmiques $(-)$; vaisseaux sanguins dilatés $(-\rightarrow$ ). CS hyperactifs (août). G $\times 400$.

figure $6 \quad$ : Dilated cells with big nuclei $(\rightarrow)$ ); intracytoplasmic granules $(-)$; dilated blood vessels (- - 1. Hyperactive CS (Auoust). G x 400.

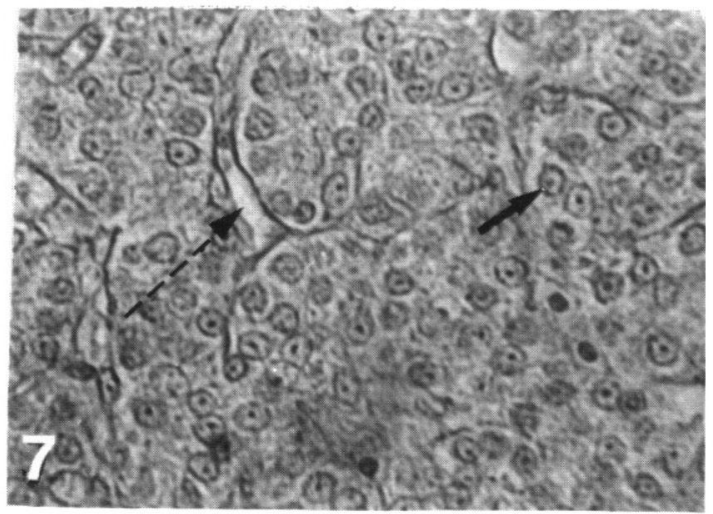

figure $7 \quad$ : Grosses cellules avec noyaux dilatés à nucléoles apparents ; cytoplasmes dégranulés $(\rightarrow$ ). Vaisseaux sanguins dilatés $(\rightarrow)$ ). CS hyperactifs (octobre). G $\times 400$.

figure $7 \quad$ : Large cells with dilated nuclei and big nucleoli ; empty cytoplasms $(\rightarrow)$. Enlarged blood vessels (.$\rightarrow$ ). Hyperactive CS (October). G X 400 . 
figs. 8, 9, 10 : Coupes transversales de CS prélevés sur des Gardons pêchés à Isbergues.

$11,12,13$ Coloration Cleveland Wolfe.

figs. 8, 9, 10 : Cross sections of CS Roach caught at the station of Isbergues.

$11,12,13$ Staining Cleveland Wolfe.

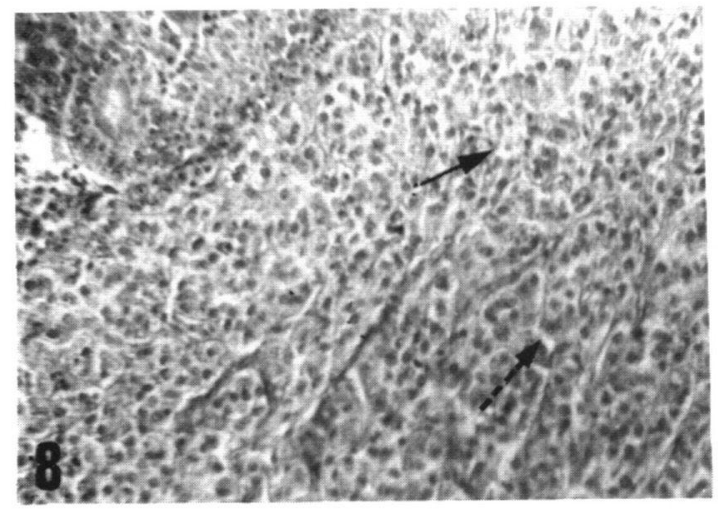

figure $8 \quad$ : Cordons cellulaires s'ètendant en larges méandres $(\rightarrow)$; vaisseaux sanguins dilatés (- - ). CS hyperactifs (août). G $\times 160$.

figure $8 \quad$ : Large cellular widening cords $(\longrightarrow)$; dilated blood vessels $(\rightarrow)$. Hyperactive CS (August). G $\times 160$.

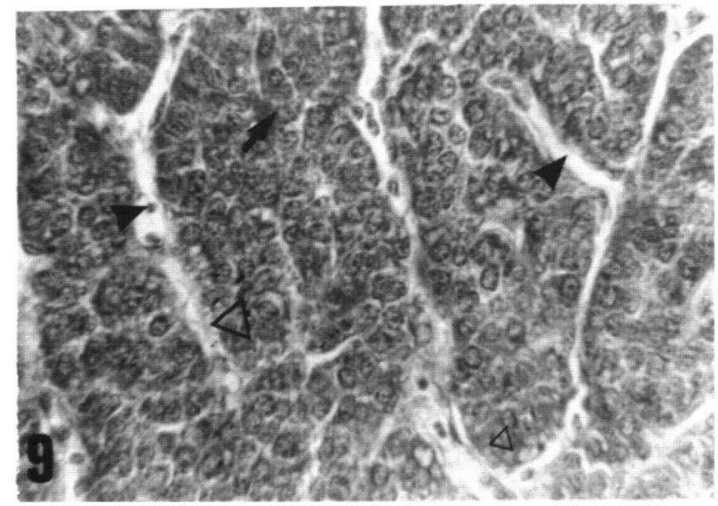

figure $9 \quad$ : Cellules pycnotiques très basophiles, sans limites cellulaires nettes $(\longrightarrow)$. Espaces de rétraction $(-)$. Noyaux pycnotiques $(D)$. CS en voie de dégénérescence après blocage de la sécrétion (août). $\mathrm{G} \times \mathbf{4 0 0}$.

figure $9 \quad$ : Pycnotic basophilic cells without visible cellular membranes $(\rightarrow)$. Retraction spaces $(-)$ ). Pycnotic nuclei $(D)$. Degenerative $C S$ after that the secretion was stopped (August). G $\mathrm{G} 400$.

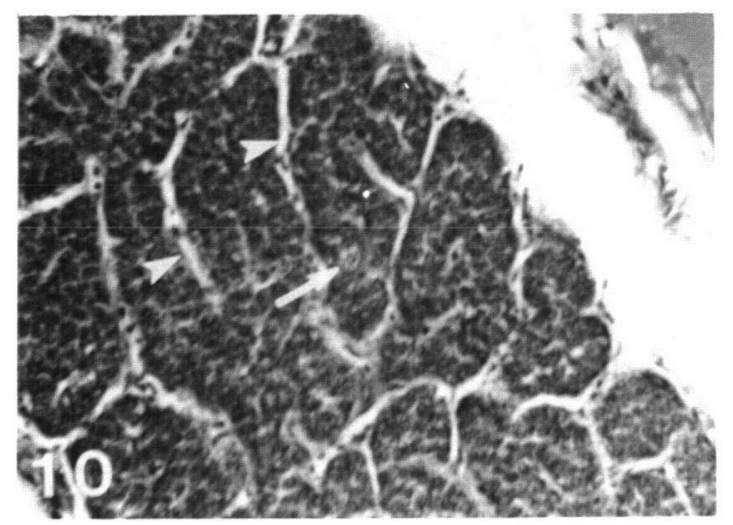

figure 10 : Cellules pycnotiques très basophiles groupées de façon anarchique en cordons denses $(\longrightarrow$ ). Espaces de rétraction ( - ). Aspect pathologique (octobre). $\mathbf{G} \times 400$.

figure 10 : Pycnotic basophilic cells, disposed out of order to form dense cords $(\rightarrow)$. Retraction spaces ( - ). Pathological aspect (October). G $\times 400$. 


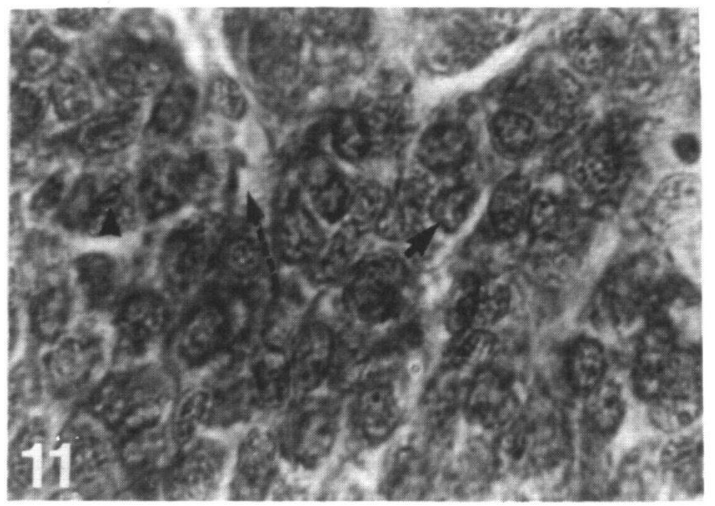

figure 11 : Cellules hypertrophiées avec très gros noyaux et plusieurs nucléoles $(\rightarrow)$; grosses granulations intracytoplasmiques $(-)$; vaisseaux sanguins très dilatés $(-)$ ). Hyperactivité pathologique (octobre). G x 400.

figure 11 : Hypertrophic cells with very big nuclei $(\rightarrow)$. Voluminous intracytoplasmic granulations $(-)$. Very dilated blood vessels. Pathologic hyperactivity (October). $\mathrm{G} \times 400$.

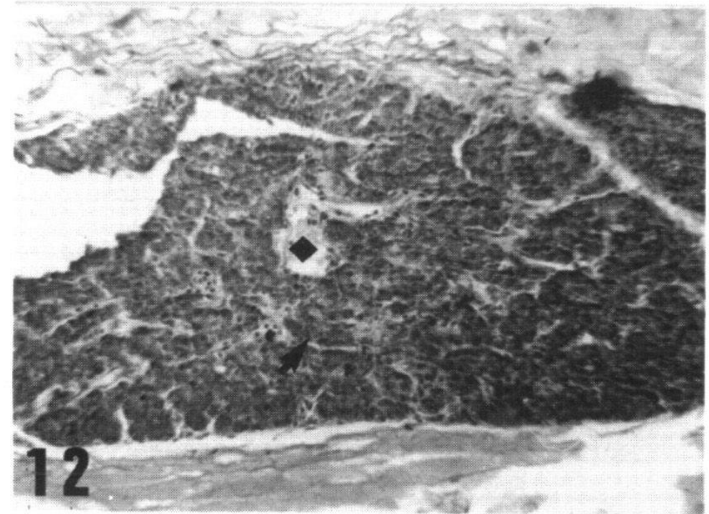

figure 12 : CS de taille très réduite présentant une atrophie notable. Travées de cellules très denses séparés par des espaces de rétraction $(\rightarrow)$. Régions en dégénérescence ( ). CS en voie de dégénérescence (mars 1987). G x 160.

figure 12 : Very small size CS with a notably atrophy. Dense cellular arches with retraction spaces (-). Degenerative area ( ). CS in a degeneration way (March 1987). G $\times 160$.

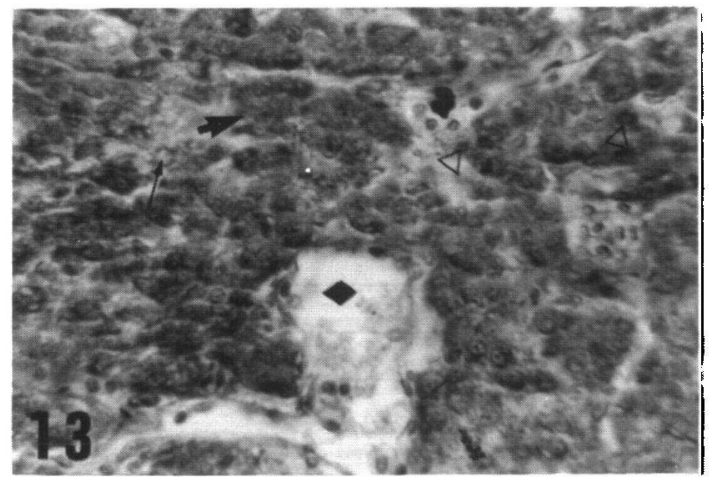

figure 13 : Cellules pycnotiques très basophiles disposées de façon anarchique $(\longrightarrow)$. Petits noyaux pycnotiques $(D)$. Région en dégénérescence $(\triangleright)$. Persistance de quelques noyaux clairs $(\rightarrow)$ ). CS en dégénérescence (mars 1987). G x 400 .

figure 13 : Very basophilic pycnotic cells disposed without of order $(\rightarrow)$. Little pycnotic nuclei $(D)$. Degenerative area $(\triangle)$. Some clear nuclei may be observed $(\longrightarrow)$. Degenerative CS (March 1987). G 4400 . 


\section{CONCLUSIONS}

La très grande sensibilité des CS à différentes modifications de l'environnement (LOPEZ et al., 1981 b; LOPEZ et al., 1985) fait que ces glandes donnent un premier signal, facilement décelable, d'un processus de dégradation physiopathologique; en conséquence ils peuvent être considérés comme de bons indicateurs d'une pollution chronique.

Cette première approche écotoxicologique, bien que partielle, et considérée comme préliminaire, nous apporte des informations non négligeables quant à l'impact de certaines modifications du milieu telles que celles qui ont pu se produire à Beuvry et plus particulièrement à Isbergues, sur certains aspects de la physiologie du Gardon.

\section{REMERCIEMENTS}

Nous remercions vivement, pour leur collaboration, M. M. GRANDMOUGIN de l'Agence de l'Eau Artois-Picardie et son équipe, ainsi que les agents du Conseil supérieur de la Pêche de Compiègne.

\section{BIBLIOGRAPHIE}

CRESPO S., SORIANO E., SAMPERA C., BALASCH J., 1981 - Zinc and copper distribution in excretory organs of the dogfish Scyliorhinus canicula and cloride cell response following treatment with zinc sulphate. Mar. Biol., 65, 117-123.

DAOUST P.Y., WOBESOR G., NEWSTEAD J.D., 1984 - Acute pathological effects of inorganic mercury and copper in gill of rainbow trout. Vet. Pathol., 21, 93-101.

FONTAINE M., 1964. - Corpuscules de Stannius et régulation ionique ( $\mathrm{Ca}, \mathrm{K}, \mathrm{Na}) \mathrm{du}$ milieu intérieur de l'Anguille (Anguilla anguilla L.). C.R. Acad. Sc. (Paris), 259, 875-878.

FONTAINE M., 1967. Intervention des corpuscules de Stannius dans l'équilibre phosphocalcique du milieu intérieur d'un poisson Téléostéen, l'Anguille. C.R. Acad. Sc. (Paris), 264, 736-737.

FONTAINE M., LOPEZ E., PEIGNOUX-DEVILLE J., 1973. Des relations existant entre l'activité de deux formations endocrines (corps ultimobranchial et corpuscules de Stannius), l'importance du squelette, la nature de la biocœnose et le régime alimentaire chez certains Scaridés (Pacifique et Méditerranée). C.R. Acad. Sc., (Paris), 276, 2.969-2.972.

HAWKES H.W., 1977. Fate and effects of petroleum hydrocarbures in marine organisms and ecosystems. Pergamon Press, Wolfe D.A. ed., p. 115-118.

KARLSSON-NÖRRGREN L., RUNN P., HAUX C., FÖRLIN L., 1985. Cadmium-induced changes in gill morphology of zebrafish. Brachydanio rerio (Hamilton-Buchanan) and rainbow trout, Salmo gairdneri Richardson. J. Fish. Biol., 27, 81-95.

KARLSSON-NÖRRGREN L., BÖRKLUND I., LJUNGBERG O., RUNN P., 1986. Acid water and aluminium exposure : experimentally induced gill lesions in brown trout, Salmo trutta $L$. J. Fish. Dis., 9, 11-25.

LELOUP-HATEY J., 1964. Modifications de l'équilibre minéral de l'anguille, Anguilla anguilla L., consécutives à l'ablation des corpuscules de Stannius. Compt. Rend. Soc. Biol., 158, 711-715.

LOPEZ E., 1969. Étude histophysiologique des corpuscules de Stannius de Salmo salar L. au cours des diverses étapes de son cycle vital. Gen. Comp. Endoc., 12, 339-349.

LOPEZ E., FONTAINE M., 1967. Réponse des corpuscules de Stannius de l'Anguille, Anguilla anguilla L., à des blessures expérimentales. Comp. Rend. Soc. Biol., 161, 36-39.

LOPEZ E., LELOUP-HATEY J., HARDY A., LALLIER F., MARTELLY E., OUDOT J., PEIGNOUXDEVILLE J., FONTAINE Y.A., 1981 a. Modifications histopathologiques et stress chez des Anguilles soumises à une exposition prolongée aux hydrocarbures. Dans "Conséquences d'une pollution accidentelle par les hydrocarbures" (645-653), C.N.E.X.O. Pub. Sci. Tech.

LOPEZ E., PEIGNOUX-DEVILLE J., LALLIER F., MARTELLY E., FONTAINE Y.A., 1981 b. Anguilles contaminées par les hydrocarbures après l'échouage de l'Amoco-Cadiz. Modifications histopathologiques des ovaires, des branchies et des glandes endocrines. C.R. Acad. Sci., (Paris), 292, 407-411.

LOPEZ E., TISSERAND-JOCHEM E.M., EYQUEM A., MILET C., HILLYARD C., LALLIER F., VIDAL B., Mac INTYRE I., 1984. Immunocytochemical detection in eel corpuscles of Stannius of a mammalian parathyroïd-like hormone. Gen. Comp. Endocr., 53, 28-36.

LOPEZ E., PEIGNOUX-DEVILLE J., VIDAL B., LALLIER F., BECKER P., 1985. Effects of WasteGypsum Disposal on some aspects of teleost fish physiology. Proceedings of the 35 th annual meeting fertilizer industry round table, $52 \mathrm{p}$. 
MALLET J., 1985. Fish gill structural changes induced by toxicants and other irritants, a statistical review. Can. J. Fish. Aquat. Sci., 42, 630-648.

MARTOJA R., TRUCHET M., LOPEZ E., LALLIER F., VIDAL B., 1984. Mise en évidence de lithiases rénales chez des poissons littoraux de la Manche et de la Mer du Nord (Limanda limanda, Platichtys flesus). Relation avec la dégénérescence des corpuscules de Stannius et les ulcérations tégumentaires. C.R. Acad. Sc. Paris, 298, 7, 185-190.

MILET C., PEIGNOUX-DEVILLE J., MARTELLY E., 1979. Gill calcium fluxes in the eel, Anguilla anguilla $L$. Effects of Stannius corpuscles and ultimobranchial body. Comp. Biochem Physiol., 61 A, 61-70.

MILET C., HILLYARD C.J., MARTELLY E., GIRGIS S., Mac INTYRE I., LOPEZ E., 1980. Similitudes structurales entre l'hormone hypocalcémiante des corpuscules de Stannius (PCS) de l'Anguille (Anguilla anguilla L.) et de l'hormone parathyroïdienne mammalienne. C.R. Acad. Sc. (Paris), 291, série D, 977-980.

MILET C., MARTELLYE., LOPEZ E., 1989. Partial purification of the parathyrin from the corpuscles of Stannius (PCS) of the eel (Anguilla anguilla L.) Gen. Comp. Endoc., 76, 83-94.

NADKARNI U.B., GORBMAN A., 1966. Structure of corpuscles of Stannius in normal and radiothyroidectomized chinook fingerlings and spawning pacific Salmon. Acta Zool., 47, 61-66.

OLIVEREAU M., 1963. Action de l'aldactone sur les corpuscules de Stannius de l'Anguille. C.R. Acad. Sci. (Paris), 257, 4.035-4.038.

OLIVEREAU M., 1964. Corpuscles of Stannius in sea-water eel. Amer. Zoologist., 4, $n^{8} 4,170$.

PEREIRA J.J., 1988. Morphological effects of mercury exposure on window-pane flounder gills as observed by scanning electron microscopy. J. Fish. Biol., 33, 571-580.

PETTIT A., 1986. Recherches sur les capsules surrénales. J. Anat. Physiol., 32, 369-419.

TISSERAND-JOCHEM E.M., LOPEZ E., MILET C., VIDAL B., MAGNAC C., EYQUEM A, COHN D.V., 1987. Co-localization and secretion of parathyrine of Stannius corpuscles (immunoreactive parathyroid hormone) and of secretory glycoproteins including secretory protein-l in the European eel (Anguilla anguilla L.). Bone and Mineral, 2, 163-174. 